\title{
Teledermatologia: correlação diagnóstica em serviço primário de saúde*
}

\author{
Teledermatology: diagnostic correlation in a primary care service
}

\author{
Cristiana Silveira Silva ${ }^{1}$ \\ Isabelle Ary Duque ${ }^{3}$ \\ Nayra Rodrigues Melo 5 \\ Paulo Ricardo Criado ${ }^{7}$
}

\author{
Murilo Barreto Souza \\ Luciana Molina de Medeiros ${ }^{4}$ \\ Cecília de Almeida Araújo ${ }^{6}$
}

\begin{abstract}
Resumo: Fundamentos: O termo telemedicina faz referência ao uso de tecnologias de comunicação para a transmissão a distância de informações relacionadas à saúde. Esse recurso é utilizado em várias especialidades médicas, principalmente naquelas em que a interpretação de imagens representa uma etapa fundamental na formulação diagnóstica.

OвJетіvo: Avaliar a concordância entre o diagnóstico presencial e o diagnóstico a distância de lesões cutâneas, utilizando a teledermatologia, em pacientes atendidos em uma unidade básica de saúde.

MÉTODOs: Foi realizado um estudo prospectivo envolvendo pacientes atendidos no serviço de dermatologia da clínica FTC em Salvador - BA. Participaram do estudo um dermatologista responsável pela consulta presencial e dois dermatologistas responsáveis pela consulta a distância. Os diagnósticos obtidos através da teleconsulta foram avaliados quanto à concordância e comparados com os diagnósticos da consulta presencial. RESULTADOS: Foram incluídos neste estudo 60 pacientes. Observou-se um grau de concordância total variando de 86,6\% a 91,6\% com índice Kappa de 0,62.

ConCLUSÃO: A teledermatologia é uma forma de assistência com um grande potencial de uso na dermatologia, podendo representar uma ferramenta útil principalmente em casos clínicos de baixa complexidade, oriundos de unidades básicas de saúde.
\end{abstract}

Palavras-chave: Consulta remota; Diagnóstico; Telemedicina

\begin{abstract}
Background: Telemedicine can be defined as the use of telecommunication technologies for the transmission of health data. It has been described in different medical specialties, especially those in which interpretation of images represents a fundamental key in formulating diagnosis.

OBJECTIVE: To evaluate the role of teledermatology in primary care system.

METHODS: A prospective analysis included 60 patients seen in a primary care unit. All patients were seen by a dermatologist as regular outpatient dermatology consultation. A medical student obtained digital images and a brief clinical history of all patients. Using a Telemedicine system these data were reviewed by two dermatologists for distance diagnosis. Agreement between the diagnoses was assessed.

RESULTS: Good agreement, ranging from $86.6 \%$ to $91.6 \%$, was achieved between direct observation and teleconsultation. Good agreement was also achieved between two telemedicine diagnosis (Kappa $=0.62)$.

CONCLUSION: Teledermatology is a form of care with great potential for use in dermatology, and could represent a useful tool in cases of low complexity from primary health units.

Keywords: Diagnosis; Remote consultation; Telemedicine
\end{abstract}

Recebido em 30.12.2008.

Aprovado pelo Conselho Consultivo e aceito para publicação em 31.07.09.

* Trabalho realizado no ambulatório de dermatologia da Faculdade de Medicina da FTC - Salvador (BA), Brasil

Conflito de interesse: Nenhum / Conflict of interest: None

Suporte financeiro: Nenhum / Financial funding: None

Médica dermatologista. Pós-graduanda nível mestrado do Hospital das Clínicas da Faculdade de Medicina da Universidade de São Paulo (HCFMUSP) São Paulo (SP), Brasil.

Pós graduando nível doutorado da Faculdade de Medicina da Universidade de São Paulo (FMUSP). Professor de informática médica da Faculdade de Tecnologia e Ciências (FTC) - Salvador (BA), Brasil.

Médica dermatologista - Fortaleza (CE), Brasil.

Médica dermatologista - São Paulo (SP), Brasil

Médica dermatologista - Teresina (PI), Brasil.

Acadêmica de medicina da FTC - Salvador (BA), Brasil

Doutor em dermatologia pela Universidade de São Paulo (USP) - São Paulo (SP), Brasil.

(C)2009 by Anais Brasileiros de Dermatologia 


\section{INTRODUÇÃO}

O termo telemedicina faz referência ao uso de tecnologias de comunicação para a transmissão a distância de informações relacionadas à saúde. ${ }^{1,2}$ Nos últimos anos um crescimento exponencial do interesse nessa área tem sido observado. O número de artigos publicados e indexados pelo Medline abordando a telemedicina passou de pouco mais de 60 , na década de 90, para aproximadamente 9.000 atualmente. ${ }^{3,4}$

A utilização da telemedicina vem sendo descrita em várias especialidades médicas, principalmente naquelas em que a interpretação de imagens representa uma etapa fundamental na formulação diagnóstica. ${ }^{5,6,7}$ A utilização deste recurso pode oferecer uma série de potenciais vantagens, principalmente em situações como as observadas no Brasil, um país com grandes dimensões geográficas e com uma distribuição irregular dos profissionais de saúde, ${ }^{8}$ especialmente dermatologistas, os quais se concentram em sua maioria em áreas de maior poder aquisitivo, como o sudeste e sul do país. Esse fato dificulta o acesso à consulta dermatológica de grupos populacionais dispersos em áreas afastadas de grandes centros. ${ }^{8}$

A dermatologia é uma especialidade com significativo componente visual, sendo um campo particularmente favorável ao uso da telemedicina.' A aplicação da teledermatologia, como comumente é denominada a utilização da telemedicina na dermatologia, vem sendo estudada principalmente como forma de assistência médica a distância, modalidade conhecida como teleconsulta., ${ }^{70,11}$

A utilização da teledermatologia para fins de consultas a distância pode ser classificada em dois grupos principais. ${ }^{5,6,71,12} \mathrm{Na}$ modalidade "store-andforward-system" (armazenamento e envio) os dados referentes à solicitação da consulta a distância, uma vez enviados, ficam armazenados em um banco de dados e são acessados após um intervalo de tempo variável. Nessa modalidade a comunicação entre os agentes envolvidos no processo ocorre de forma assincrônica. Na modalidade "real-time" (tempo real), a troca de informações e a interação entre os agentes ocorrem em tempo real. ${ }^{8,12}$

O objetivo deste estudo é avaliar a concordância entre o diagnóstico presencial e o diagnóstico a distância de lesões cutâneas, utilizando a teledermatologia, nos pacientes atendidos em uma unidade básica de saúde, por meio do método de armazenamento e envio (SFS).

\section{MATERIAL E MÉTODOS}

Após aprovação pela Comissão de Ética em Pesquisa, foi realizado um estudo prospectivo envolvendo pacientes atendidos no ambulatório de dermatologia da Faculdade de Medicina da FTC, Salvador -
BA, entre janeiro e abril de 2007. Para possibilitar o envio e a recepção de dados a distância foi criado um ambiente na internet, utilizando ASP (Active Server Pages), acoplado ao banco de dados Microsoft SQL Server $^{\mathrm{TM}}$.

Participaram do estudo um dermatologista responsável pela consulta presencial em Salvador - BA e dois dermatologistas responsáveis pela consulta a ser respondida a distância (teleconsulta), que se localizavam em duas capitais brasileiras distintas: São Paulo SP e Fortaleza - CE.

Todos os pacientes que foram atendidos no ambulatório de dermatologia da Faculdade de Medicina da FTC, Salvador - BA, entre janeiro e abril de 2007, foram convidados a participar do estudo. Antecedendo a consulta presencial, e após a assinatura do termo de consentimento pós-informado, um estudante de medicina foi responsável por colher a história clínica e fotografar as lesões. Juntamente com as fotos, os seguintes dados foram coletados e posteriormente enviados para a teleconsulta: sexo, idade, queixa principal, história da moléstia atual de forma resumida, tempo de duração do quadro em questão e descrição de características elementares do exame físico. Precedendo o início do estudo, o estudante de medicina foi instruído sobre a forma adequada de fotografar e descrever as lesões observadas. Foram incluídos no estudo apenas pacientes que apresentassem lesões dermatológicas visíveis.

As fotografias digitais das lesões cutâneas foram adquiridas sob iluminação artificial (luz fria), em consultório médico, utilizando-se a câmera digital Sony Cyber-Shot ${ }^{\mathrm{TM}}$ com resolução de 7.2 megapixels. Foram obtidas em média 10 fotos por paciente, incluindo fotos panorâmicas e no modo "macro". Após essa captura inicial, foram selecionadas três a quatro fotos de melhor qualidade, que foram enviadas para a teleconsulta.

As imagens foram transferidas da câmera para o computador através de cabo USB e armazenadas no formato JPEG (Joint Photographic Experts Group), com resolução de $3.072 \times 2.304$ pixels.

Para o envio dos dados referentes a cada paciente, foi utilizado um ambiente na internet criado exclusivamente para esse fim. Sempre que um novo caso era incluído, uma mensagem era enviada via correio eletrônico para os médicos participantes, informando que havia uma nova solicitação de consulta a distância. Através de um acesso restrito os médicos responsáveis pela teleconsulta obtinham os dados referentes a cada solicitação de consulta.

Após acessar os dados, cada médico responsável pela teleconsulta fornecia o seu diagnóstico de forma independente. Todos os médicos formularam 
seus diagnósticos separadamente com base nas informações clínicas fornecidas e nas fotografias. Além do diagnóstico, também era possível o envio de comentários sobre os casos, com o objetivo de obter informações e críticas que pudessem identificar as dificuldades enfrentadas em cada caso.

Após a coleta dos dados, foram avaliadas as falhas e dificuldades do funcionamento do sistema e o grau de concordância entre os diagnósticos clínicos dos médicos envolvidos no estudo. O diagnóstico considerado de referência foi aquele feito pelo médico responsável pelo atendimento presencial. Foi considerado como totalmente concordante (TC) o diagnóstico a distância descrito de forma idêntica ao realizado pelo médico responsável pelo atendimento presencial. Foi considerado como parcialmente concordante (PC) o diagnóstico fornecido pelo médico a distância que correspondia a um diagnóstico diferencial daquele fornecido inicialmente na consulta presencial, e foi considerado como diagnóstico discordante (DD) o diagnóstico via teleconsulta que diferia do diagnóstico principal e dos diagnósticos diferenciais fornecidos pelo médico responsável pela consulta presencial.

Neste estudo foi adotado um nível de significância de 5\%. A concordância entre os acertos diagnósticos dos dermatologistas responsáveis pela teleconsulta foi avaliada a partir do teste Kappa. A análise estatística foi realizada utilizando o software BioEstat $5.0^{\mathrm{TM}}$.

\section{RESULTADOS}

Foram incluídos neste projeto piloto 60 pacientes. A idade dos pacientes variou entre 14 e 62 anos, sendo $44 \%$ do sexo feminino e $56 \%$ do sexo masculino. A lista com os diagnósticos obtidos durante a consulta presencial está exposta na tabela 1 .

Além do diagnóstico presencial, para cada paciente foram gerados dois diagnósticos principais, fornecidos por cada um dos médicos responsáveis pela teleconsulta, tendo sido obtidas no total 120 respostas.

O grau de concordância total entre os diagnósticos obtidos através da teleconsulta e os obtidos na consulta presencial variou de $86,6 \%$ a $91,6 \%$ (Gráfico 1). Agrupando os diagnósticos considerados como TC e PC, a concordância variou entre 96\% e 100\% (Gráfico 1). Considerando apenas os diagnósticos obtidos através da teleconsulta, foi observada uma boa concordância entre os dermatologistas responsáveis pela consulta a distância (Kappa $=0,62, \mathrm{p}<0,01)$.

Em relação aos comentários obtidos, das 120 respostas, a má qualidade da foto foi relatada em 20 $(16,6 \%)$, e a necessidade de dados adicionais para uma conclusão diagnóstica foi relatada em 24 (20\%). A insuficiência de dados foi referente à necessidade de informações extras, como exame anatomopatológico, exame dermatoscópico e/ou dados referentes à descrição da consistência ou detalhes da textura da pele.

\section{DISCUSSÃO}

Para a utilização da telemedicina na modalidade teleconsulta, existem duas formas principais de intercâmbio de dados: "store-and-forward" - quando as informações transmitidas ficam armazenadas até serem acessadas - e "real-time" - quando ocorre a interação em tempo real entre as partes envolvidas. ${ }^{4,7,9}$

Apesar de a modalidade em tempo real apresentar vantagens quando comparada com a modalidade SFS, como uma maior interatividade entre o paciente e o médico e um menor tempo de resposta, dificuldades como a maior complexidade, o custo mais elevado e a necessidade de um acesso sincrônico entre os agentes envolvidos para a troca de informações favorecem a modalidade SFS. ${ }^{8,12}$ Por conta dessas características, a modalidade SFS foi adotada neste estudo.

A taxa de concordância total referida na literatura médica varia entre $54 \%$ e $95 \%,{ }^{13,14}$ e a concordância parcial varia entre $76 \%$ e $100 \% .{ }^{12}$ Neste trabalho o grau de concordância total entre o diagnóstico presencial e a distância foi considerado alto, variando de $86,6 \%$ a $91,6 \%$. Quando considerada a concordância parcial, esse número se elevou para 98,3\%-100\%. Esse alto grau de concordância entre os diagnósticos presencial e a distância e a baixa taxa de discordância entre os médicos responsáveis pela consulta a distância podem ser explicados pelo fato de os casos serem oriundos de um serviço de atendimento básico de saúde, representando, em sua grande maioria, casos

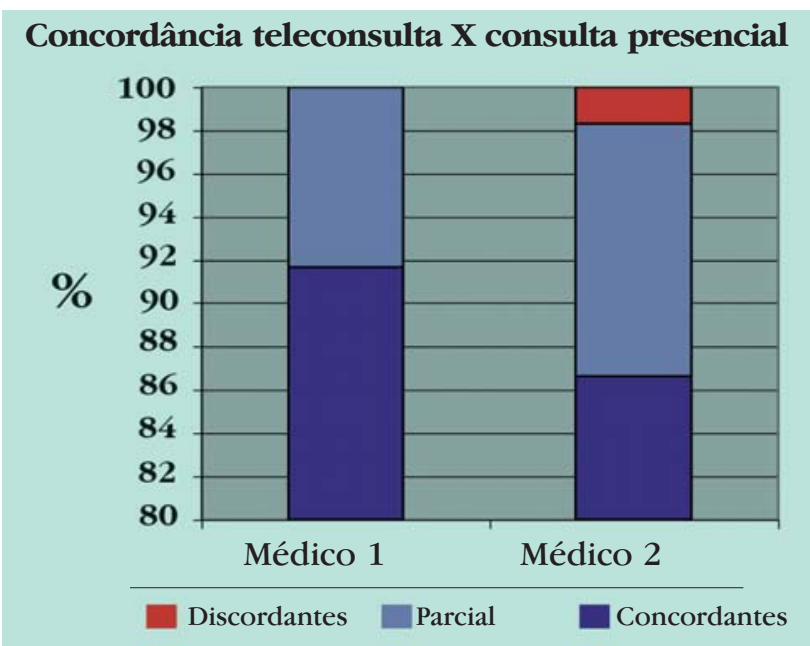

GrÁfICo 1: Grau de concordância total (Concordantes), concordância parcial (Parcial) e discordância (Discordantes) entre os diagnósticos obtidos via consulta presencial e via teleconsulta 
TABela 1: Lista dos diagnósticos de cada paciente de acordo com a consulta presencial

\begin{tabular}{|c|c|c|c|}
\hline Caso & Diagnóstico & Caso & Diagnóstico \\
\hline 1 & Dermatite perioral & 31 & Candidose intertriginosa \\
\hline 2 & Impetigo & 32 & Siringoma \\
\hline 3 & Hidradenite supurativa & 33 & Pitiríase versicolor \\
\hline 4 & Alopecia areata & 34 & Psoríase do couro cabeludo \\
\hline 5 & Lúpus discoide & 35 & Nevo melanocítico congênito \\
\hline 6 & Molusco contagioso & & $36 \quad$ Nevo azul \\
\hline 7 & Granuloma anular & 37 & Herpes simples \\
\hline 8 & Xantelasma & 38 & Herpes zóster \\
\hline 9 & Ceratose actínica & 39 & Dermatite ocre \\
\hline 10 & Dermatofibroma & 40 & Pápulas podais piezogênicas \\
\hline 11 & Eritema fixo medicamentoso & 41 & Pseudoacantose nigricante \\
\hline 12 & Eczema atópico & 42 & Esclerodermia em placas \\
\hline 13 & Líquen simples crônico & 43 & Líquen plano \\
\hline 14 & Acne vulgar & 44 & Líquen amiloidótico \\
\hline 15 & Dermatite de contato & 45 & Líquen escleroso \\
\hline 16 & Estrias atróficas & 46 & Cisto mucoso digital \\
\hline 17 & Vitiligo & 47 & Mastocitose \\
\hline 18 & Verruga plantar & 48 & Lentigo maligno melanoma \\
\hline 19 & Tinha do couro cabeludo & 49 & Condiloma acuminado \\
\hline 20 & Psoríase vulgar & 50 & Eritema nodoso \\
\hline 21 & Onicomicose & 51 & Eritema polimorfo \\
\hline 22 & Carcinoma basocelular & 52 & Queratoacantoma \\
\hline 23 & Dermatose papulosa nigra & 53 & Pitiríase rósea \\
\hline 24 & Acne ocupacional (cloracne) & 54 & Miliária rubra \\
\hline 25 & Queratólise plantar sulcada & 55 & Urticária \\
\hline 26 & Foliculite queloidiana da nuca & 56 & Hiperceratose neviforme dos mamilos \\
\hline 27 & Celulite na face & 57 & Dermatite seborreica \\
\hline 28 & Nevus flammeus & 58 & Rosácea \\
\hline 29 & Poiquilodermia solar & 59 & Síndrome de Reiter \\
\hline 30 & Escabiose & 60 & Melasma \\
\hline
\end{tabular}

de baixa complexidade e com apresentação típica. Essa associação entre um índice de acerto elevado e a baixa complexidade do caso clínico já foi evidenciada em outros estudos. ${ }^{15}$ Outro fator que pode ter influenciado o grau de concordância é o fato de todos os dermatologistas envolvidos no trabalho apresentarem formação e experiência similar na prática dermatológica.

Em relação à segurança e funcionamento do sistema utilizado para a transmissão e armazenamento dos dados, não foi observado qualquer tipo de falha. Tampouco foi observado qualquer tipo de dificuldade de manejo com a interface criada, tendo sido seu uso assimilado com grande facilidade por todos os participantes do estudo.

A qualidade técnica das fotos influencia de forma decisiva no grau de acerto diagnóstico e concordância entre observadores. ${ }^{8,12}$ Para minimizar problemas com a qualidade das fotos, foram tiradas em média 10 fotos por paciente, incluindo fotos panorâ- micas e no modo "macro", e dentre elas foram selecionadas três a quatro fotos com melhor qualidade, considerando o foco e a iluminação, para serem enviadas pelo sistema. Mesmo com esse cuidado, a queixa de má qualidade da foto foi identificada em 16,6\% dos casos. Essa queixa foi mais frequente em fotos que identificavam regiões como pavilhão auricular e couro cabeludo, já comumente consideradas de difícil visualização. $^{12}$

Neste estudo, com o objetivo de simular informações obtidas por um profissional da área da saúde não especialista em dermatologia, as informações utilizadas para a solicitação da teleconsulta foram obtidas por um estudante de medicina. O desempenho do sistema neste estudo sugere a possibilidade de que, com um treinamento adequado, um profissional não especialista em dermatologia possa ser o responsável pela coleta das informações a serem utilizadas para a solicitação da teleconsulta, com a manutenção de resultados satisfatórios. 


\section{CONCLUSÃO}

A teledermatologia assistencial com auxílio da internet é uma das formas mais difundidas de teleassistência no mundo, representando uma opção viável devido ao baixo custo e à simplicidade tecnológica para a sua aplicação. ${ }^{13}$

Casos de baixa complexidade ou oriundos de unidades básicas de saúde podem ser particularmente beneficiados pelos sistemas de teledermatologia. ${ }^{13}$
A telemedicina é um recurso válido para melhorar a cobertura de saúde,${ }^{13}$ principalmente em um país de dimensões continentais como o Brasil. As aplicações e possibilidades da teledermatologia são múltiplas; ${ }^{8}$ restam, porém, vários desafios, principalmente no que diz respeito a aspectos éticos e legais do atendimento médico a distância. ${ }^{13}$

\section{REFERÊNCIAS}

1. Wootton R. Telemedicine. BMJ. 2001;323:557-60.

2. Perednia DA, Brown NA. Teledermatology: one application of telemedicine. Bull Med Libr Assoc. 1995:83:42-7.

3. Demartines N, Mutter D, Vix M, Leroy J, Glatz D, Rösel $\mathrm{F}$, et al. Assessment of Telemedicine in Surgical Education and Patient Care. Ann Surg. 2000;231:282-91.

4. Romero G, Cortina P, Vera E. Telemedicina y teledermatologia (II): estado actual de la investigación en teleconsulta dermatológica. Actas Dermosifiliogr. 2008;99:586-97.

5. Eedy DJ, Wootton R. Teledermatology: a review. Br J Dermatol. 2001:144:696-707.

6. D'Elia PB, Fisher PD, Bordin R, Harzheim E, Ramos MC. Concordância entre diagnósticos dermatológicos feitos presencialmente e por imagens digitais. An Bras Dermatol. 2007;82:521-7.

7. Perednia DA, Brown NA. Teledermatology: one application of telemedicine. Bull Med Libr Assoc. 1995:83:42-7.

8. Miot HA. Desenvolvimento e sistematização da interconsulta dermatológica à distância [tese]. São Paulo (SP): Universidade de São Paulo; 2005. 144 p.

9. Romero G, Garrido JA, García-Arpa M. Telemedicine and teledermatology (I): concepts and applications. Actas Dermosifiliogr 2008;99:506-22.

10. Taylor P, Goldsmith P, Murray K, Harris D, Barkley A. Evaluating a telemedicine system to assist in the management of dermatology referrals. Br J Dermatol. 2001:144:328-33.

11. Whited JD. Teledermatology research review. Int J Dermatol. 2006:45:220-9.

12. Miot HA, Paixão MP, Wen CL. Teledermatologia: passado, presente e futuro. An Bras Dermatol. 2005;80:523-32.

13. Wootton R, Bloomer SE, Corbett R, Eedy DJ, Hicks N, Lotery HE, et al. Multicentre randomised control trial comparing real time dermatology with conventional outpatient dermatological care: societal cost-benefit analysis. BMJ. 2000;320:1252-6.

14. Chao LW, Cestari TF, Bakos L, Oliveira MR, Miot HA, Zampese $\mathrm{M}$, et al. Evaluation of an Internet-based teledermatology system. J Telemed Telecare. 2003;9 (Suppl1):S9-12.

15. Vidmar DA, Cruess D, Hsieh $P$, Dolecek $Q$, Pak H, Gwynn M, et al. The effect of decreasing digital image resolution on teledermatology diagnosis. Telemed J. 1999;5:375-83.

ENDEREÇO PARA CORRESPONDÊNCIA / MAILING ADDRESS:
Cristiana Silveira Silva
Rua da Graça, $n^{\circ} 374$. Bairro Graça
40080410 Salvador BA
Tel.:/fax: 7133362850
e-mail: silveira.cristiana@gmail.com

ENDEREÇO PARA CORRESPONDÊNCIA / MAILING ADDRESS:

Cristiana Silveira Silva

Rua da Graça, 374. Bairro Graça

Tel.:/fax: 7133362850

e-mail: silveira.cristiana@gmail.com

Como citar este artigo/How to cite this article: Silva CS, Souza MB, Duque IA, Medeiros LM, Melo NR, Araújo CA, Criado PR. Teledermatologia: correlação diagnóstica em serviço primário de saúde. An Bras Dermatol. 2009;84(5):489-93. 